cephradine (given as $11 \mu \mathrm{g} / \mathrm{ml}$ after a 500 $\mathrm{mg}$ oral dose) readily exceeds the M.I.C. in all cases and exceeds the M.B.C. for all except the methicillin-resistant strains. These results are far more persuasive than those quoted in the promotional literature.-I am, etc.,

Westminster Medical School

SYDNEY SELWYN London S.W.1.

SIR,-While accepting that it is the duty of experts like Drs. J. D. Williams and A. M. Geddes to keep a watchful eye on the advertising of antibiotics, I would like to refute the implications of that part of their letter (14 April, p. 116) dealing with the advertising of amoxycillin. It is my opinion that this letter may itself mislead doctors into believing that amoxycillin and ampicillin are the same compound, which is completely untrue.

Amoxycillin is absorbed, distributed in the body, and eventually excreted from the body as amoxycillin and at no time is it converted to ampicillin. As is correctly stated, the two antibiotics differ in structure only by an OH group but, as any pharmacologist can confirm, this can make a radical change in the properties of a drug. One only has to consider L-dopa, which differs from L-tyrosine merely by the possession of an $\mathrm{OH}$ group, and the remarkable difference in properties between benzylpenicillin and ampicillin resulting from the substitution of an $\mathrm{NH}_{2}$ group.

Certainly amoxycillin and ampicillin have a similar (but not identical) antibacterial activity. Amoxycillin, however, has been shown to be more effective in experiments in vivo and this has recently been shown to be associated with higher bactericidal activity against many Gram-negative organisms (G. N. Robinson, to be published). The absorption of the two antibiotics is quite different. May ${ }^{1}$ has shown that some patients with infections that have failed to respond to ampicillin have, indeed, been improved by amoxycillin with conversion of purulent sputum to mucoid.

Finally, the two antibiotics differ in the frequency with which they induce rashes in patients with infectious mononucleosis. Whereas ampicillin causes rash in about $90 \%$ of patients suffering from this disease, a search of our records of over 5,000 patients treated with amoxycillin reveals seven patients with an eventual diagnosis of infectious mononucleosis, only two of whom developed a rash. Although it is too early to draw any definite conclusions, our present information suggests that the incidence of rash is less than that of ampicillin and more in line with that of other oral antibiotics.

Of course amoxycillin is closely related to ampicillin, but therapeutic progress frequently results from a close study of the differences between two related drugs rather than from overstating their similarities. This is one of the directions, therefore, that we shall continue to explore for new and more effective antibiotics.-I am, etc.,

E. T. KNUDSEN, Medical Director
Beecham Pharmaceutical

Brentford, Middlesex 1 May, J. R., and Ingold, A., British Foumal of
Serum Lithium Estimations

SIR,-Dr. J. G. Weir (10 February, p. 356) must have been unfortunate with his serum lithium determinations. The disparity which he quotes between simultaneous determinations in different laboratories $(0.6$ and 1.0 $\mathrm{mEq} / 1 ., 0.2$ and $0.46 \mathrm{mEq} / 1$.) is considerably larger than that found in parallel determinations in most laboratories. With a reasonably sensitive flame photometer it is not difficult to achieve relative standard deviations of $2 \%$ or less. (For those who use Eppendorf flame photometers it may be worth knowing that photomultiplier RCA $931 \mathrm{~A}$ is more sensitive at $671 \mathrm{~nm}$ than the previously recommended photomultiplier RCA IP 22).

Dr. Weir also reports that the serum lithium concentration might vary considerably from time to time even in patients who meticulously maintained constant lithium intakes. In one of his patients the serum lithium level was usually around $0.4-0.6 \mathrm{mEq} / 1$., it then suddenly rose to $2.0 \mathrm{mEq} / 1$., and a fortnight later it was $0.2-0.5 \mathrm{mEq} / 1$, all with a constant lithium intake. This variation may of course have been due to analytical error, but it seems unlikely. If the blood samples were drawn at different time intervals after the intake of lithium-and nothing to the contrary appears from Dr. Weir's letter-variation was in fact to be expected.

The variation has two main causes, one during the first 10 to 12 hours after the intake of lithium and another thereafter. During the hours following the intake of lithium, when the lithium ion is being absorbed from the gastrointestinal tract, the serum lithium concentrations shows marked variations (fig. 1), and since the rate of absorption differs from one person to another (fig. 2) as well as in the same person from day to day serum lithium concentrations during the period of absorption are ill-suited as guides for treatment. With ordinary lithium tablets complete gastrointestinal absorption may last 6 to 8

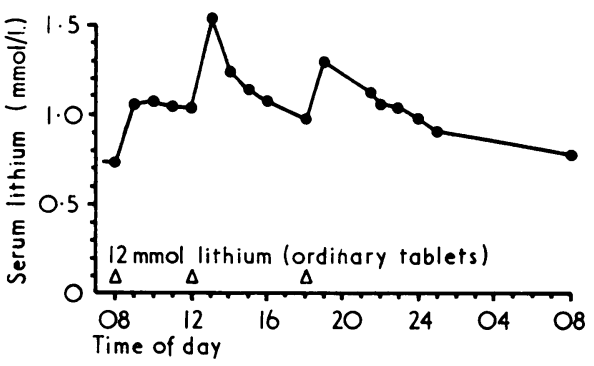

FIG. 1-Serum lithium concentrations throughout 24 hours in a patient on lithium maintenance treatmen with drug administrations at 8 a.m., 12 noon, and 6 p.m.

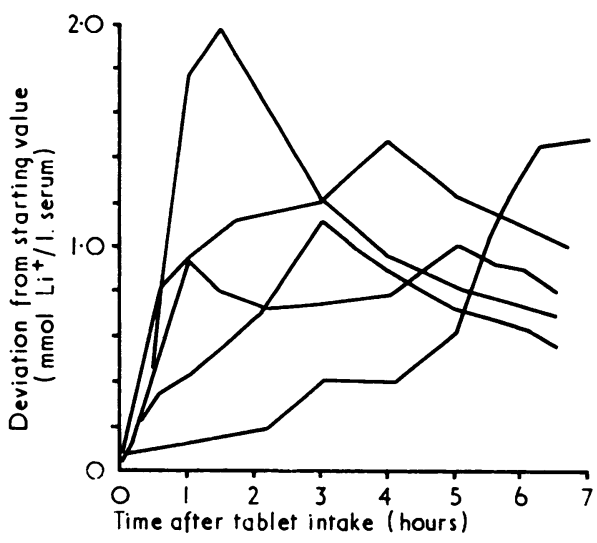

FIG. 2-Changes in the serum lithium concentration of five patients given a single dose of lithium at time zero. hours and with retard preparations 8 to 10 hours. Blood samples drawn for monitoring purposes should accordingly not be drawn earlier than at least 10 hours after the last intake of lithium -perhaps, to be on the safe side, 12 hours would be better.

When gastrointestinal absorption of lithium is finished the serum lithium concentration falls evenly and the fall follows an exponential course. Blood samples drawn, for example, 18 or 24 hours after the last intake will therefore show lower lithium values than blood samples drawn at 12 hours. So far as Dr. Weir's patient is concerned it seems possible that the serum lithium values of $0.2 \longrightarrow 0.5 \mathrm{mEq} / 1$., and $0.4-$ $0.6 \mathrm{mEq} / 1$, were found in blood samples drawn, for example, 18 hours after the last intake of lithium and that the value of $2.0 \mathrm{mEq} / 1$., was found in a sample drawn before the gastrointestinal absorption had been finished.

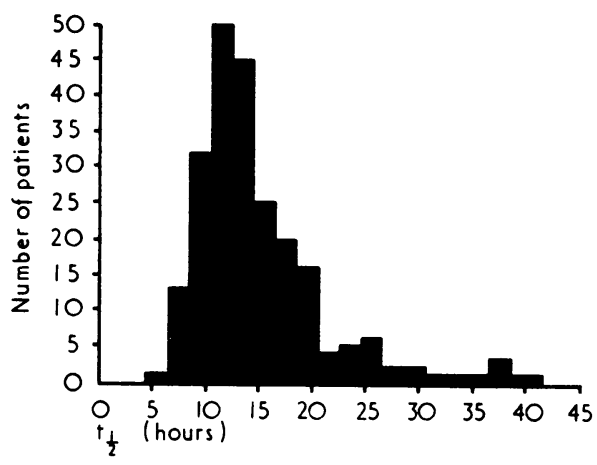

FIG. 3-Distribution of 226 patients according to their serum lithium half-time, $t \frac{1}{2}$, during the intake of lithium.

The rate at which the serum lithium concentration falls after absorption is finished is determined primarily by the renal lithium clearance. Since this varies considerably from one person to another the rate at which serum lithium falls also shows great variation (fig. 3), and consequently one cannot employ a common factor for converting 18-hour or 24-hour values to 12-hour values. This means that standardization of the time interval between the last intake of lithium and the drawing of blood is essential when using serum lithium concentrations as a reliable guide for treatment and adjustment of dosages. A time interval of 12 hours seems recommendable for both theoretical and practical reasons. Employment of the term "12-hr standard serum lithium" might serve to emphasize the importance of having the time interval standardized.-I am, etc.

AMDI AMDISEN

The Psychopharmacological Research Unit, 8240 Risskov, Denmark

\section{Acute Pancreatitis in Mycoplasma pneumoniae Infections}

SIR,-Several complications of respiratory tract infections with Mycoplasma pneumoniae have been described, including meningoencephalitis, pericarditis, and various skin manifestations. ${ }^{12} \mathrm{We}$ have recently seen four patients (aged from 15 to 69 years) with acute pancreatitis after pneumonia caused by $M$. pneumoniae. In three of the patients the pancreatitis developed in the third week after the onset of cough, by which time the respiratory tract symptoms had almost disappeared.

The diagnosis of $M$. pneumoniae infection 
was based on a significant rise in the titres rate during the phases of resting respiration, of complement-fixation and metabolisminhibiting antibodies to the organism. Cold agglutinins were present in all cases. In two of them agglutination also occurred in blood samples kept at room temperature. All the patients had high serum levels of IgM $(330-500 \mathrm{mg} / 100 \mathrm{ml})$, while the concentrations of IgG and IgA were normal. Such a selective increase in IgM is known to occur in infections caused by mycoplasmas. ${ }^{34}$ All the patients had raised serum and urine concentrations of amylase. Analysis of isoenzymes of amylase in serum suggested that the increase in this enzyme in the serum originated from the pancreas. None of the patients had a history of gall stone disease and none took alcohol. No virus was isolated from throat washings and faeces and serological tests for coxsackie B, cytomegalo, and mumps virus were also negative.

Three of the four patients had a severely depressed respiratory exchange and two of them had to be treated with a respirator. One patient had E.C.G. changes of the type seen in pericarditis. One of them developed a hyperosmolar diabetic coma one week before she died. She had a high titre of cold agglutinins and multiple thromboses were found at necropsy. The three patients who survived had an increased concentration of serum amylase for several weeks after the clinical symptoms of pancreatitis had disappeared. Two of them had a transient rise in the blood glucose level.

A relationship between infectious pancreatitis, such as in mumps ${ }^{5}$ and coxsackie B infections, ${ }^{6-8}$ and diabetes mellitus has been suggested. In this respect pancreatitis in $\boldsymbol{M}$. pneumoniae infections requires further investigation.-We are, etc.,

\section{P.-A. MÅRDH} B. URSING

Department of Infectious Diseases,

University Hospital,
Lund, Sweden

1 Sterner, G., and Biberfeld, G., Scandinavian 2 Gournal of Infectious Direases, 1969, 1, 203. Grayston, J. T., Foy, H. M., and Kenny, G. E.,
in The Mycoplasmatales and the L-phase of in The Mycoplasmatales and the L-phase of North-Holland Publishing Company, 1969.

Feizi, T., Annals of Ner

Sciences, 1967, 143, 801.

Mardh, P.-A., Acta Pathologica
Scandinavica, 1970, 78b, 726 . Melin, K., and M. G., Bolton, R., and Taylor, K. W. W., British Medica! fournal, 1969, 3. 627.
Gamble, D. R., and Taylor, K. W., British

Medical fournal, 1969, 3, 631.
Gamble, D. R., and Taylor, K. W., British Medical foumal, 1973, 1, 289.

\section{Effect of Respiration on Parkinsonian} Tremor

SIR,-A tremor artefact on the electrocardiogram is a now well-recognized feature of Parkinson's disease. The accompanying figure shows such an artefact varying with the phases of resting respiration-an observation which to my knowledge has not been recorded previously.

In this case quiet inspiration (centre of record) was associated with a marked reduction of the gross tremor, which returned during the period of expiration. Although there was no obvious change in the heart
I wonder if the decrease in vagal tone which is believed to occur during inspiration contributed its own anticholinergic effect which reduced the tremor? Since inspiration requires the positive contraction of the respiratory muscles it is possible that this effort alone could have been sufficient to reduce the tremor, especially since resting expiration is mainly the result of elastic forces. However, the presence of a greater tremor artefact during the period of forced expiration at the end of the E.C.G. record would appear to contradict this latter suggestion.-I am, etc.,

King's College Hospital,

BRIAN LivesLey

\section{Radioimmunossay Follow-up of Hydatidiform Mole}

SIR,-The letters of Sir John Stallworthy (3 March, p. 550) and Professor W. S. Tow (7 April, p. 49) seem to be answered by the data in my letter (17 February, p. 414). Repetition is justified only to counter the tendency to rate data from Singapore as more relevant to patients in the United Kingdom than data collected here. Professor Tow refers to a Singapore "malignancy rate" of $36.6 \%$ in women aged 40 or more, whereas in our series of 280 patients followed up after hydatidiform mole the "malignancy rate" for women aged 15 to 54 was $5.7 \%$ and for those aged 32 o. more it was $0 \%$. Singapore and the U.K. might differ in their disease patterns, or in their criteria of malignancy, or in both these factors. Extrapolation from one to the other has its limitations.

I see no objection to primary hysterectomy for the elderly or multiparous woman provided the follow-up be as careful as without hysterectomy. The suggestion that I am rash to claim that hysterectomy would not reduce the mortality rate is intriguing, since the rate was zero without routine hysterectomy in the series under discussion. However, if hysterectomy is life-saving in Singapore, why is it not advocated routinely for young and old alike? Either a life-saving procedure is withheld so that the survivors preserve their reproductive function or its benefits are judged to be marginal. What is the current rate of exchange between saving life and saving reproductive function and who fixes it?

Unfortunately, the place of hysterectomy is unlikeiy to be defined by the "scientifically designed prospective study" advocated by Sir John. It is a first principle to compare like with like. Our data showed that comparing hysterectomized elderly patients with non-hysterectomized younger patients, as he suggests, would be fruitless. Instead, patients would have to be defined by age and parity and allocated at random to hysterectomy and non-hysterectomy groups. Since we have seen choriocarcinoma presenting up to 17 years after primary hysterectomy for mole, the follow-up would need to be protracted. Such a trial might be difficult to organize, but the recently introduced registration scheme for patients with hydatidiform mole should go some ways to providing more ex- tensive data than have been available hitherto.-I am, etc.,

Charing Cross Hospital (Fulham)

K. D. BAgshawE

London W.6

\section{Anaesthetic Contamination}

SIR,-I read with interest your leading article on anaesthetic contamination of operating theatres (24 March, p. 693) and the article by Dr. R. S. Vaughan and others on its prevention (p. 727). While I congratulate Dr. Vaughan and his colleagues on their valuable contribution to this subject I would like to point out that the solution offered by them solves only part of this problem. The activated charcoal filter will remove only the vapours of volatile anaesthetics, still leaving anaesthetic gases such as nitrous oxide and cyclopropane to pollute the operating theatre environment.

Nitrous oxide is a major cause of pollution in operating theatres, perhaps because it is used in high flow and concentration. In one study nitrous oxide has been measured in end-tidal samples and found to average 20 p.p.m. ${ }^{1}$ and another study showed that the level of nitrous oxide could be as high as 7,000 p.p.m. in the inhalation zone of anaesthetists using nonrebreathing systems. ${ }^{2}$ Chronic exposure to nitrous oxide is known to cause bone marrow depression. ${ }^{3}$ Bruce et al., ${ }^{4}$ in their survey of causes of death in anaesthetists over the period 1947-66, concluded that the incidence of lymphoid and reticuloendothelial malignancy was two to three times greater than expected, amounting to almost $25 \%$ of all deaths due to malignant causes. Bruce Johnson $^{5}$ suggests that nitrous oxide may be the causative agent of these malignant conditions and possibly of other problems associated with the operating room. In a recent study my associates and I have shown that nitrous oxide and cyclopropane in clinical concentrations inhibit the growth of respiratory pathogens and that this inhibition is related to the time of exposure rather than to the concentrations of anaesthetic gases (unpublished observations). The antimitotic effect of nitrous oxide has been demonstrated in studies on cultures of mouse myoblasts and on the growth of cells in monolayer culture. ${ }^{78}$ It may be that the anaesthetic gases do cause abnormalities of cell division, and nitrous oxide may be responsible for congenital abnormality, spontaneous abortion, and involuntary infertility.

In a study now in progress Dr. W. J. Cole and $I$ have found a high concentration of isopropyl alcohol vapour along with the anaesthetic agents causing contamination of the environment. In all air samples taken from the operating theatre this consistently produced a most intense peak on the gas chromatograph trace which was observed after the halothane peak. A $70 \%$ solution of isopropyl alcohol with chlorhexidine is commonly used for the preparation of skin before surgery. With the help of an Askrog exhaust valve we have been able to eliminate effectively the anaesthetic gases and vapours from the operating theatres. In the air samples from the theatre where the system is in use the concentration of halothane vapour has been found to be less than 0.1 p.p.m. We consider that venting of anaesthetic gases and vapours from the theatre is a simple, effective, and inexpensive method 